\title{
Produkt- und Technologieentwicklungen für eine nachhaltige Energieversorgung
}

\author{
Nicht nur die Klimakrise und die Begrenztheit \\ fossiler Rohstoffe stellt Energieversorgungs- \\ unternehmen vor erhebliche Herausforderun- \\ gen. Um zu einer nachhaltigen Energieversor- \\ gung beizutragen, sind verschiedene Ansätze \\ notwendig. \\ Von Heinrich Tschochohei und Andrea Köhler
}

I sich die regenerative Energieerzeugung durchsetzt.

I eine umweltschonende Mobilität (beispielsweise Fahrzeuge als Stromspeicher) zum Einsatz kommt.

Vor diesem Hintergrund verbindet EWE seine drei Geschäftsbereiche Energie, Infrastruktur und Kommunikationstechnologie kontinuierlich konsequent miteinander. Bezogen auf die drei Aspekte von Nachhaltigkeitsstrategien seien nachfolgend einige Beispiele genannt, wie sich dieser Ansatz in Inventionen und Innovationen, differenziert nach Gerlach, niederschlägt (Gerlach 2008).

\section{Effizienzstrategie}

Nachfolgend soll dokumentiert werden, inwieweit sich der Anspruch eines Energiedienstleisters mit der Anforderung eines verminderten Energieeinsatzes kombinieren lässt. Aus der Angebotsperspektive ist beispielsweise das EWE-Engagement für die Weiterentwicklung der Brennstoffzellentechnologie zu nennen; hier engagiert sich das Unternehmen bei Feldversuchen zu Kleinst-Kraft-Wärme-Kopplung (EWE AG 2009b).

Nachfrageseitig soll die Art und Weise des Energieeinsatzes und der Energieproduktion durch interaktive Verfahren der Energiebereitstellung und -messung gefördert werden. Dazu testet EWE die „EWE Box“ zurzeit in 400 Haushalten der Region Oldenburg. Mit der EWE Box sollen Verbraucher in die Lage versetzt werden,

I aktuelle Verbrauchswerte direkt zu ermitteln.

I Verbräuche zu analysieren und mit Referenzhaushalten zu vergleichen.

I das eigene Verbrauchsverhalten direkt mit Kostengrößen in Verbindung zu bringen.

I in Verbindung mit spezifischen Produkten zusätzlich Energie zu sparen.

Letztlich stellt sich aber bei Umwelt- und Klimaschutz nicht nur die Frage, welche Technologien Lösungsmöglichkeiten bieten, sondern auch, wie Informations- und Motivationsbarrieren zielgruppenadäquat überwunden werden können (Schmidt/Tschochohei 2007). Vor diesem Hintergrund hat die EWE AG 2007 das Zentrum Zukunft errichtet. Hier werden innovative Ansätze, die eine effiziente Energieversorgung mit modernster Informationstechnologie verbinden, einem breiten Publikum vorgestellt. In Ausstellungen zu Heiz- und Klimatechnik, zur Solarenergie und zum Wohnen, beispielsweise in einem komplett eingerichteten Wohnbereich, erleben Verbraucher und Multiplikatoren die Möglichkeiten intelligenter Verbrauchssteuerung. 


\section{Konsistenzstrategie}

Kopfmüller beschreibt die Konsistenzstrategie im Kern einer Substitutionsstrategie (Kopfmüller 2001). Danach sei unter anderem eine Ersetzung des mit Nachhaltigkeitszielen unverträglichen Verbrauchs fossiler Energieträger durch regenerative Energieträger anzustreben.

Neben langjährigen Engagements auf technologischer Seite, wie zum Beispiel im Rahmen von alpha ventus, dem ersten deutschen Offshore-Windpark in der Nordsee, möchte sich EWE durch innovative Endkundenprodukte positionieren.

Eines davon, der EWE Werder Strom, strebt an, die Umwandlung von Sonnenenergie in Strom erfahrbar zu machen und gleichzeitig positive Emotionen auszulösen. Auf dem Bremer Weser-Stadion befindet sich Deutschlands größte gebäudeintegrierte Fotovoltaik-Anlage. Der hier generierte Strom gehört zum regenerativen Strommix, der im Rahmen dieses Angebots vermarktet wird. Das Angebot richtet sich insbesondere an Fußballfans, die besondere, an die Ergebnisse ihres Clubs gekoppelte Tarifstrukturen angeboten bekommen. Auf diese Weise kann es gelingen, neue Kundengruppen für Strom aus erneuerbaren Energiequellen zu begeistern.

\section{Suffizienzstrategie}

Zahrnt betont, dass eine Prioritätensetzung in der Energiewirtschaft lauten müsste: „Zuerst kommt Energie einsparen, dann die Energieeffizienz und schließlich die erneuerbaren Energien“ (Zahrnt 2007). EWE nimmt die Energieeinsparung in der Weise ernst, als dass für Gewerbe- und Privatkunden über mehrere Jahre eine Bandbreite an Dienstleistungs- und Beratungsangeboten entwickelt wurde, die den Verbraucher bei der Energieeinsparung unterstützen. Potenziale für die Energieeinsparung bietet beispielsweise die Sanierung unzureichend wärmegedämmter Gebäude (EWE AG 2009c). Durch Energieeinsparmaßnahmen im Altbaubestand könnten 50 bis 80 Prozent des Energieverbrauchs eingespart werden.

Pläne und Förderprogramme zur Energieeinsparung bleiben wirkungslos, wenn sich Endverbraucher, aus Gründen wie Informations- oder Motivationsmangel, nicht beteiligen. Wie Barrieren im Nutzerverhalten durch anreizorientierte Mechanismen überwunden werden können, zeigt das Modellprojekt „Reduktionszertifikate im Haushalt", das EWE gemeinsam mit dem Landkreis Emsland ins Leben gerufen hat. Rund 170 Einund Mehrfamilienhäuser nehmen daran teil. Seit 2007 wird für die Dauer von drei Jahren untersucht, wie der Kohlenstoffdioxid-Ausstoß dieser Gebäude durch einen neuen erdgasbetriebenen Heizkessel, den Einsatz erneuerbarer Energien, durch Modernisierung, Sanierung oder ein verändertes Verbrauchsverhalten der Bewohner verringert werden kann.

Die Teilnehmer werden anfangs von Spezialisten intensiv beraten. Für jede Tonne Kohlenstoffdioxid, die sie zusätzlich, das heißt über autonome Minderungstrends hinaus, einsparen, erhalten sie eine Prämie.
Als konkreten Nutzen erfährt der Kunde Effekte auf informatorischer, motivationaler und ökonomischer Ebene. Der Kunde gewinnt neues Wissen und erfährt den direkten Zusammenhang zwischen Verhalten und Energieverbrauch. Schließlich wird die jährliche Erdgasrechnung um die ausgezahlte Prämie vermindert. Damit können sich geringinvestive Einsparmaßnahmen in kürzester Frist amortisieren. Auf diese Weise wird dieser Ansatz auch für Mieter interessant, die wegen des Nutzer-Investor-Dilemmas seltener in den Genuss hoch-investiver Einsparmaßnahmen kommen.

EWE versorgt damit eine Vielzahl von Menschen mit den elementaren Grundlagen für das tägliche Leben, für Wohlstand und für gesellschaftliche Entwicklung. Das Unternehmen nimmt diese Aufgabe bewusst an, um erstens eine langfristig stabile Unternehmens- und Geschäftsentwicklung zu erreichen und zweitens einen positiven Beitrag des Unternehmens zur nachhaltigen Entwicklung der gesamten Gesellschaft sicherzustellen.

\section{Literatur}

Deutscher Bundestag (Hrsg.): Nachhaltige Energieversorgung unter den Bedingungen der Globalisierung und Liberalisierung, Berlin 2002.

EWE AG (Hrsg.): 10 Bullensee-Thesen und abgeleitete Handlungsempfehlungen zur zukünftigen Energieversorgung. Oldenburg 2009a.

EWE AG (Hrsg.): Weiterführende Überlegungen zur dezentralen Mikro-KraftWärme-Kopplung. Oldenburg 2009b.

EWE AG (Hrsg.): Weiterführende Überlegungen zur Energieeinsparung. Oldenburg 2009c.

Gerlach, A.: Entscheidungsdefekte als Barrieren für Nachhaltigkeitsinnovationen. Ansätze zur Identifikation, Erklärung und Überwindung. München 2008.

Huber, J.: Nachhaltige Entwicklung. Berlin 1995

Kopfmüller, J.: Nachhaltige Entwicklung im Energiebereich. Karlsruhe 2001.

Kopfmüller, J. / Coenen, R. / Jörissen, J.: Konkretisierung und Operationalisierung des Leitbildes einer nachhaltigen Entwicklung für den Energiebereich. Karlsruhe 2000 .

Schmidt, S. / Tschochohei, H.: Soziale und ökologische Verantwortung in der Erlebnisgesellschaft. Chancen und Risiken nachhaltiger Events. In: Müller, M. / Schaltegger, S. (Hrsg.): Corporate Social Responsibility. Trend oder Modeerscheinung? München 2007, S. 175 - 192.

Zahrnt, A.: Effizienz gerne! Aber Suffizienz!, in: Rudolph, S. (Hrsg.): Wachstum, Wachstum über alles. Marburg: 2007, S. 193 - 196.

\section{- AUTOREN + KONTAKT}

Dr. Heinrich Tschochohei ist Leiter und Andrea Köhlerist Referentin im Team „CO $\mathrm{CO}_{2}$-Solutions“ in der Abteilung Vertrieb Energie der EWE AG.

EWE AG, Team $\mathrm{CO}_{2}$-Solutions. Tel.: +49 $441803-1382$ Fax: +49 441 803-1389, E-Mail: andrea.koehler@ewe.de 
(c) 20I0 Authors; licensee IÖW and oekom verlag. This is an article distributed under the terms of the Creative Commons Attribution Non-Commercial No Derivates License (http://creativecommons.org/licenses/by-nc-nd/3.o/), which permits unrestricted use, distribution, and reproduction in any medium, provided the original work is properly cited. 